\title{
Une étude des évolutions pédagogiques et didactiques dans les colloques CETSIS
}

\author{
Jean-Philippe Georget ${ }^{1}$, Jean-François Thémines ${ }^{2}$ \\ jean-philippe.georget@unicaen.fr, jean-francois.themines@unicaen.fr \\ (1) Normandie Université, CERSE EA 965, (2) Normandie Université, ESO UMR 6590
}

\begin{abstract}
RÉSUMÉ : Des enseignants et chercheurs de l'enseignement supérieur en électronique, électrotechnique et automatique se réunissent en colloques pour échanger sur leurs pratiques pédagogiques. Les organisateurs du colloque CETSIS 2013 ont " commandé » une expertise de cette pratique communautaire à des chercheurs en didactiques. Nous rendons compte de la construction de cette expertise et de ses résultats. Les colloques atteignent l'objectif qui leur est fixé, d'être un lieu de diffusion, d'échange d'expériences et de compétences. Ils tendent aussi à poursuivre implicitement d'autres objectifs. Une partie des enquêtés est désireuse de s’orienter vers des pratiques de recherche en didactique et en pédagogie dans le domaine EEA. Le morcellement du domaine en petites spécialités montre la richesse et la difficulté de cette orientation. Nous suggérons quelques développements de nature à soutenir cette orientation et ainsi compléter la richesse des colloques CETSIS.
\end{abstract}

Mots clés : pratiques pédagogiques, pédagogie universitaire, didactique, innovation, communauté de pratique.

\section{INTRODUCTION}

L'enquête que nous présentons a pour objet d'analyser le contenu pédagogique et didactique des colloques CETSIS. Elle répond à une demande d' « expertise » adressée par le Club EEA : les colloques CETSIS remplissent-ils les objectifs de partage et d'amélioration des pratiques des enseignants du domaine, notamment par la communication d'innovations didactiques et pédagogiques?

Notre point de vue est externe au domaine EEA, mais il s'inscrit dans le champ des didactiques, disciplines attachées à l'étude des phénomènes de transmission et d'appropriation de contenus. Dans le cadre de la demande qui nous était faite, nous avons voulu savoir si les colloques CETSIS constituent un outil d'innovation pédagogique, au sens où ils permettent à la communauté de ses participants d'avancer dans l'élaboration d'une pédagogie et d'une didactique universitaire propre à leurs domaines.

A partir de l'analyse des Actes de 2001 et de 2011, complétée de celle d'un questionnaire et d'entretiens réalisés en 2013 [1], nous montrons que la fonction bien installée de partage de pratiques débouche aujourd'hui sur l'émergence d'un champ de recherche spécifique dans la pédagogie universitaire du domaine. Les indices récoltés en ce sens nous amènent à quelques suggestions pour soutenir cette émergence.

\section{CHAMPS THÉORIQUES DE RÉFÉRENCE}

Notre étude se rattache à deux approches de l'activité d'enseignement et de recherche dans le supérieur. Le champ de la pédagogie universitaire [2] a d'abord développé des connaissances sur les étudiants (performances, insertion professionnelle, styles d'apprentissage, motivation). Mais la direction de recherche dans laquelle nous situons notre étude concerne le contexte académique. Nous cherchons en effet à caractériser la capacité d'un acteur universitaire collectif à faire évoluer ses pratiques ainsi que ses connaissances pédagogiques et didactiques. Cette direction de recherche est promise à se développer dans le contexte d'approfondissement des démarches d'évaluation tant à l'échelon français (2002 : circulaire d'application du schéma LMD, 2007 : création de l'AERES, 2013 : Loi sur l'Enseignement supérieur et la Recherche) que dans le cadre de l'Espace Européen de l'Enseignement Supérieur (EEES).

L’étude peut être rapprochée de l'analyse des réseaux [3] parce qu'elle cherche à savoir si des connexions se font entre membres participants du colloque, d'une édition à l'autre, aboutissant à des évolutions d'ensemble dans les références et dans les pratiques pédagogiques.

\section{PROBLÉMATIQUE ET MÉTHODOLOGIE}

\subsection{Axes de questionnement}

Le premier axe de notre questionnement concerne les aspects didactiques et pédagogiques présents dans les contributions aux actes. Quelles sont les qualités didactiques et pédagogiques mises en évidence ? En particulier, quelles sont les méthodes d'évaluation utilisées par les enseignants pour évaluer l'efficacité de leurs pratiques? Quelles sont les approches de l'apprentissage à l'œuvre ?

Le deuxième axe de questionnement concerne la capitalisation des pratiques et des réflexions relatives à ces pratiques. Y a-t-il des traces observables d'une telle capitalisation des pratiques, en quelque sorte une mémoire didactique de la communauté des participants aux colloques CETSIS [4] ?

Le troisième axe de questionnement prolonge les deux premiers, il concerne l'aspect communautaire des colloques CETSIS. Les participants aux colloques CETSIS constituent-ils une communauté ou des communautés ? Et de quelle nature ? Par exemple, peut-on dire qu'émergent des communautés de pratique [5] caractérisées par des "pratiques pédagogiques » particulières ? 


\subsection{Méthodologie}

Nous avons choisi trois moyens d'enquête [1] :

- une étude des actes des colloques CETSIS de 2001 (94 contributions) et de 2011 (84 contributions) ;

- une enquête par questionnaire auprès des participants du colloque CETSIS de mars 2013 (46 répondants, soit un inscrit sur deux) ;

- une enquête par des entretiens auprès de participants du colloque CETSIS 2013.

La présente contribution s'appuie avant tout sur l'étude des actes et l'enquête par questionnaire.

\subsubsection{L'étude des actes de colloques}

L'étude de chaque contribution vise à répondre aux questions qui se posent selon chaque axe :

- Axe «didactique et pédagogique ». Quels sont les types de tâches données aux étudiants [6] ? Quelle est l'activité attendue d'eux? Comment sont conçues ces tâches et activité : des caractéristiques et/ou des besoins d'étudiants sont-ils pris en compte, en relation avec les contenus à transmettre? Quelle évaluation pédagogique et didactique est proposée de l'activité ainsi que des dispositifs?

- Axe «capitalisation de pratiques» et axe " communautés ». Est-il fait référence aux actes antérieurs des colloques CETSIS, notamment à d'autres auteurs ? Observe-t-on des évolutions dans ces pratiques de référencement ?

\subsubsection{Le questionnaire aux participants du colloque CETSIS 2013}

Les questions ont été établies en fonction des mêmes axes :

- Axe « didactique et pédagogique » : Avez-vous déjà modifié un TP ou un cours en relation avec une présentation vue à un colloque CETSIS ? Si oui, comment qualifieriez-vous la modification introduite : aménagement mineur, adaptation substantielle, transformation radicale, etc. ?

- Axe «capitalisation de pratiques »: Si vous avez déjà mis en œuvre un TP ou un projet présenté à un colloque, s'agit-il d'un TP ou d'un projet dont vous avez vu la présentation ? Si oui, avez-vous personnellement rencontré l'auteur après sa présentation? Si vous avez déjà contribué aux actes, avez-vous réutilisé une contribution d'un autre auteur des actes CETSIS (comme point de départ, comme inspiration...), etc. ?

- Axe «communautés». Quelles sont les raisons principales de votre fréquentation des colloques CETSIS : partager des pratiques, trouver des pratiques nouvelles à expérimenter, partager des réflexions sur les évolutions de l'enseignement, retrouver les collègues spécialistes du domaine, montrer vos essais, diversifier votre pratique de recherche vers la pédagogie ou la didactique dans votre spécialité ?

\section{RESULTATS}

\subsection{Les actes : une sensibilité croissante aux questions didactiques}

La comparaison des contributions entre 2001 et 2011 fait apparaître une continuité dans les formes et les contenus des contributions, exceptions faites des aspects technologiques propres au champ EEA. Dans le format attendu (6 pages maximum) la structure des articles est relativement homogène. Pour une grande majorité, il s'agit de la présentation d'outils ou de dispositifs d'enseignement. La communication fait se succéder la présentation du matériel conçu pour, adapté, ou développé en vue de la transmission de contenus, l'adéquation technique de l'outil à l'enseignement de ces contenus, la narration des manipulations possibles ou réalisées. Quelquefois, cette présentation est précédée d'un exposé des raisons de la mise en place de l'outil ou du dispositif. Le plus souvent, elle s'ouvre en conclusion sur un rapide bilan de satisfaction et des perspectives de développement du matériel.

Les tâches à réaliser par les étudiants renvoient majoritairement à des gestes techniques. Les objectifs d'apprentissage ne sont pas toujours explicités : les contenus disciplinaires tiennent lieu d'objectif pédagogique. Les mentions de prise en compte de besoins d'étudiants, en relation avec les difficultés intrinsèques de contenu ainsi qu'avec la provenance et la diversité de ces étudiants, demeurent minoritaires : 26 communications sur 84 en 2011. De plus, les auteurs ne communiquent généralement pas sur les outils qui leur permettent d'établir ces besoins. Fréquemment, ils affichent leur souhait de résoudre des problèmes de motivation ou d'intérêt des étudiants dans certaines disciplines ou par rapport à des contenus.

Plus rares sont les indices de méthodes d'évaluation critériée et objectivable qui dépassent des impressions générales ressenties par les auteurs : «bilan positif ", «bon investissement des étudiants ». Le thème de la notation des travaux des étudiants n'est pratiquement jamais évoquée (1 communication sur 84 en 2011) de même que l'identification et l'évaluation du degré de maîtrise des compétences construites par les étudiants. Les retours d'expérience, terme le plus usité, se limitent à la mention de ressentis déclarés. Ce constat peut paraître surprenant car les entretiens tendent à montrer que la question de l'évaluation, notamment dans le cadre des démarche(s) projet, est justement un sujet complexe qui préoccupe les participants.

Une différence significative apparaît entre les deux éditions. Le nombre d'expérimentations situées dans le cadre de projets passe de 16 en 2001 à 38 en 201. La diversité prévaut, tant pour le statut du projet (projet tutoré, encadré, intensif, mini-projet, démarche de projet) que pour sa définition (de l'implicite à l'expression des fondements pédagogiques de la démarche). Une certaine homogénéité marque cependant la description fort peu précise des 
interactions professeurs-étudiants ou étudiantsétudiants durant ces projets.

Parmi les 38 communications qui évoquent en 2011 un cadre de projet, 13 affichent des conceptions ou des convictions précises en matière d'apprentissage. Quatre peuvent être distinguées : l'Apprentissage par Problèmes et par Projets, la créativité, la gestion de projets à référence industrielle, les méthodes de pédagogie active. L'étude des références bibliographiques ne permet pas d'affirmer qu'il y ait un effet de capitalisation lié aux colloques. Les différents auteurs s'auto-réfèrent lorsqu'ils ont participé à plusieurs colloques, à l'exception de certains des tenants de l'Apprentissage par Problèmes et par projets qui s'appuient sur des références diverses: revue scientifique de psychologie cognitive, journal professionnel du supérieur, productions propres.

L'analyse des actes tendrait donc à montrer l'émergence de préoccupations didactiques " pratiques ». Une certaine fragilité est à noter. D’une part, quelques petites équipes, plutôt enclines à travailler ces questions, ont tendance à s'auto-référer. D'autre part, la dimension apprentissage est généralement très peu documentée dans l'analyse $a$ priori des tâches comme dans l'évaluation de l'activité des étudiants. Autrement dit, ce qui est appris, le comment et le pourquoi c'est appris restent souvent implicites dans les contributions analysées.

\section{2 Les questionnaires : la possibilité de voir émerger une activité de recherche didactique}

L'analyse des réponses aux questionnaires (46, soit 1 inscrit sur 2) conforte le résultat obtenu par l'étude des actes.

Conformément aux objectifs de l'association organisatrice, les participants de l'édition du colloque 2013 déclarent avant tout venir partager des pratiques (40 sur 46 répondants). 28 déclarent venir pour partager des réflexions sur les évolutions de l'enseignement universitaire, ce qui témoigne d'un souci de s'inscrire dans une réflexion plus large que celle qui concerne le domaine EAA. 13 affirment que les colloques CETSIS ont des effets sur leurs pratiques alors même que 25 des 46 répondants sont des primoparticipants.

Par ailleurs, 13 répondants sur 46 déclarent venir au colloque pour diversifier leur pratique de recherche vers la didactique ou la pédagogie dans leur spécialité. Pour certains d'entre eux, cela semble même être un objectif majeur de leur participation. De plus, parmi ces 13 personnes, 7 sont des primo-participants et ils semblent donc se saisir du colloque comme d'un lieu de partage de questions ou de résultats de recherche.

En somme, alors que l'étude des actes montre une sensibilité croissante aux questions didactiques, sans que n'apparaisse une maîtrise experte du champ de référence, l'étude des questionnaires viendrait asseoir l'idée d'une émergence notable de la didactique ou de la pédagogie comme activité de recherche dans le domaine EEA.

\section{DISCUSSION}

\subsection{Transposition didactique incomplète et attachement disciplinaire fort : l'innovation technique prime}

L'activité des colloques CETSIS montre une volonté générale de s'inscrire dans les buts du Club EEA. Les colloques atteignent l'objectif qui leur est fixé, d'être un lieu de diffusion, d'échange d'expériences et de compétences pour les enseignants des formations du supérieur dans le domaine [7]. A travers l'intention de valorisation de l'enseignement supérieur [7], ce sont aussi un certain nombre de filières, de disciplines et de débouchés professionnels dont on saisit que les participants veulent les rendre plus attractifs.

Il faut cependant noter que, même si le Club EEA est ouvert à l'ensemble des acteurs enseignants, la thématique de la liaison secondaire-supérieur ne fait pour l'instant qu'émerger [8]. Or, elle est importante en ce qui concerne la motivation des élèves et des étudiants, thème auquel les intervenants sont sensibles. Mais elle l'est aussi pour la prise en compte des parcours de formation, des progressions d'apprentissage, des compétences numériques préexistantes, dans l'élaboration des matériels et des dispositifs pédagogiques du supérieur.

L'objectif de diffusion et d'échanges d'expériences et de compétences rencontre pourtant des limites. Le " quoi enseigner » l'emporte très nettement sur le « comment enseigner » ainsi que sur le pourquoi et le pour qui. La préface des Actes de 2001 affirme que "l'Homme en tant que Sujet doit avoir une place dans l'objectivité des études scientifiques » [7]. Très peu de communications appréhendent cependant cette dimension de la formation des étudiants dans le domaine. De même, la démarche qualité, laquelle intègre l'évaluation des enseignements par les étudiants ainsi que celle des organismes recruteurs, fait l'objet d'un tout petit nombre de présentations, malgré l’actualité institutionnelle de la question.

C'est donc le «quoi enseigner » qui domine, en relation soit avec les évolutions professionnelles, techniques et organisationnelles, soit avec les reconfigurations des filières et les changements de programmes. La prise en compte des contraintes de temps, de programme et de coût des installations l'emporte sur celles de variables didactiques tant du côté des étudiants (diversité, représentations préalables, motivation, pratiques sociales) que des situations mise en place (degré de guidage de l'activité, ressources mises à disposition, interactions prévues et effectives, etc.). Le constat qui prime est l'absence d'analyse micro-didactique, c'est-à-dire le niveau de la séquence d'enseignement ou de la séance d'enseignement. Le domaine didactique est davantage présent par l'échelle macro- (les évolutions curriculaires) ou meso- (les changements organisationnels dans un département).

Une première raison possible pour expliquer cette absence de l'approche micro-didactique est le format : 
4 à 6 pages permettent difficilement de présenter un dispositif technique complexe, son analyse du versant " sciences de l'ingénieur " (analyse du savoir savant et de son implémentation dans un dispositif, un matériel, un outil) suivie d'une analyse du versant didactique et pédagogique.

Ainsi, la transposition didactique des savoirs [9] n'est analysée que partiellement. Par exemple, les limites des simulations proposées pour la formation ne sont pas toujours évoquées ; les usages effectifs qu'en ont les étudiants ne sont que très rarement décrits. Ceci ne signifie pas pour autant que les auteurs ne s'en soucient pas, comme le montrent certains entretiens menés avec des auteurs de contributions, mais cela peut paraître surprenant quant il s'agit de l'enseignement des sciences de l'ingénieur.

Une deuxième raison possible est l'attachement des contributeurs à l'aspect disciplinaire de leurs expérimentations. En effet, les TP proposés sont fréquemment novateurs par le fait qu'ils sont en phase avec les innovations technologiques du monde professionnel, voire parce qu'ils anticipent la généralisation d'usages émergents dans les entreprises. Ces TP incorporent une description de l'aspect disciplinaire d'autant plus nécessaire qu'ils se situent à l'intersection de différentes matières ou spécialités et développent des applications spécifiques. Leur valeur scientifique disciplinaire doit par conséquent être testée sous plusieurs aspects.

En somme, ces TP présentent toujours un degré d'innovation technique et disciplinaire, voire des considérations sur le coût financier du dispositif didactique, mais il s'agit rarement d'améliorer de façon mesurable les capacités des étudiants à apprendre mieux ou plus. La nécessité d'adaptation aux exigences industrielles contraint à l'évolution permanente des « savoirs/techniques " et des dispositifs d'enseignement (ex. salle blanche, projet de construction, projet de séances). En plus des contraintes liées au format des actes, cette adaptation continue laisse sans doute peu de place à une réflexion instrumentée en termes didactiques et pédagogiques.

\subsection{Une image du métier d'enseignant du supérieur dans le domaine, du « cour " à ses marges}

A partir de ces éléments (une transposition didactique incomplètement analysée, un aspect d'innovation technique dominant), on peut aborder le thème du métier d'enseignant du supérieur ou d'enseignantchercheur dans le domaine EEA. On rappellera qu'un métier se définit par un ensemble de gestes professionnels caractéristiques, une histoire collective dont personne n'est propriétaire mais à laquelle chacun contribue, et une définition de la tâche, de la mission confiée, des finalités de la profession [10]. L'attachement à l'innovation technique, à la scientificité disciplinaire des dispositifs et des matériels d'enseignement est constitutif du métier partagé par les participants aux colloques CETSIS. Au-delà de ce «cœur de métier » apparent, les questions de la motivation des élèves, de l'attractivité des filières, du développement de compétences autres que strictement techniques chez les étudiants, apparaissent comme des préoccupations partagées, plus qu'elles ne font l'objet d'un travail aussi systématique que le « cœur de métier ». A la marge du métier tel qu'il apparaît dans les actes, mais sans doute pas dans des lieux de discussion plus informels, figurent :

- l'évaluation, et plus précisément encore, la notation des étudiants ;

- la valorisation de leur créativité ;

- leur préparation à des fonctions de transmission de savoirs qu'il s'agisse du monde des entreprises ou de l'enseignement supérieur ;

- la description fine et l'analyse de l'efficacité des situations de formation ;

- voire la discussion professionnelle sur les finalités des formations : quel étudiant-type cherche-t-on à former, quelles qualités cherche-t-on à développer chez lui ?

La forte progression des communications qui se rapportent à des dispositifs de projet confirme cette structure du métier visible dans les actes, tout en y apportant un nouvel équilibre. Le " cœur de métier » ne se déplace pas, mais les préoccupations pour les parcours de formation, la prise en compte des aspirations des étudiants, l'expérimentation réfléchie du travail d'équipe et de la transmission d'information, se sont nettement affirmées. Des communications ciblent des compétences autres que strictement disciplinaires: le rapport à l'évaluation des étudiants (cas de l'orthographe), la prise en charge de la transposition didactique en direction d'élèves du primaire, de façon à expérimenter des situations de transmission, la conduite de projet d'ingénierie didactique (réalisation d'un outil-ressource grand public).

Les finalités des formations sont nettement exprimées dans les communications les plus explicites sur les stratégies d'enseignement. Elles forment un « archipel » dont les actes ne permettent pas, à l'heure actuelle, qu'elles dialoguent et structurent le débat professionnel. Le potentiel de débat, tributaire de la différence des conceptions en présence, est là. Il existe, comme nous l'avons dit, quatre traditions ou cultures autour des apprentissages et du projet (didactique, créative, industrielle et pédagogique). Certainement gagneraient-elles - et le métier lui-même - à dialoguer de manière formelle, dans la perspective d'un étayage plus scientifique de leur valeur formative.

\section{3 Condition d'un fonctionnement en communauté de recherche en didactique et en pédagogie dans son domaine}

Un bon tiers des répondants aux questionnaires participe à l'édition 2013 du colloque CETSIS avec une intention de diversifier sa pratique de recherche vers l'enseignement. A la lecture des Actes précédents, on peut penser qu'il n'y trouvera que de façon 
périphérique, matière à réflexion ou à persévérer dans cette intention.

Ce jugement doit être tempéré : la communication de pratiques dans les actes ne révèle pas forcément les pratiques effectives de réflexivité sur le métier ou de recherche bibliographique en rapport avec la pédagogie et la didactique de sa discipline. Cette communication peut, en dépit d'une tonalité dominante disciplinaire, suggérer aux auditeurs des pistes de recherche didactique ou pédagogique, par rapprochement d'expériences ou de lectures. Mais une question se pose: ces individus ont-ils trouvé dans l'édition CETSIS 2013 et trouveront-ils dans les éditions ultérieures un lieu adapté à cet objectif ? Plus largement, à quelles conditions une partie de la communauté des participants aux colloques CETSIS peut-elle aussi fonctionner en communauté(s) de recherche en didactique et en pédagogie dans son domaine (cf. figure 1) ?

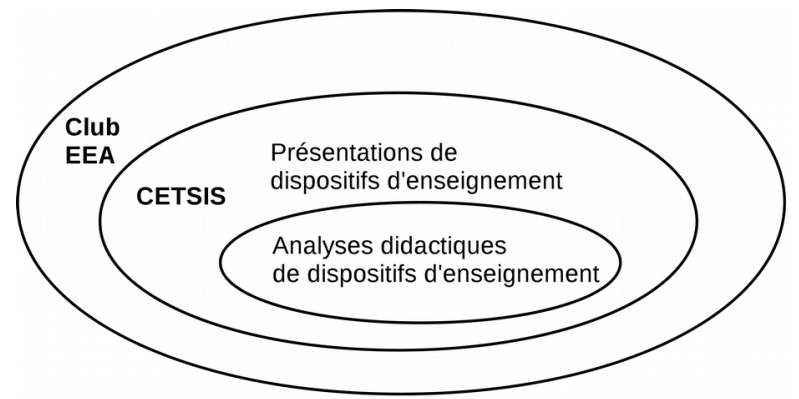

Fig. 1 : Une partie de la « communauté » CETSIS comme communauté de recherche en didactique et en pédagogie du domaine EEA.

Une première condition serait que les différences apparentes liées à la multiplicité des spécialités et matières représentées dans les colloques CETSIS soient exploitées à des fins de comparaison des dispositifs, des situations d'enseignementapprentissage, de l'implication des étudiants, etc. Une seconde condition serait de questionner des points communs jugés " évidents » (la faible motivation des étudiants, leur attrait pour le « concret », etc.) et d'en faire ainsi les points d'entrée pour des démarches de recherche.

\section{CONCLUSION ET PERSPECTIVES}

La communauté EEA, au travers des colloques CETSIS, s'est dotée d'un outil de professionnalisation de ses membres, en tant qu'individu enseignant d'une part et en tant que membre d'un collectif d'autre part [11]. Pour autant, cette professionnalisation peut sembler incomplète : le métier apparaît centré sur une innovation technique dans les contenus des matériels, outils et dispositifs d'enseignement, au détriment de tâches qui apparaissent peut-être plus banales et ingrates, mais qui sont centrales pour remplir les missions et répondre aux finalités de formation auxquels sont attachés les participants : la mesure de l'efficacité de l'enseignement, la mise en place d'une démarche qualité, l'évaluation des étudiants, l'étayage de compétences collectives, etc.

L'étude des actes montre des continuités dans la forme et les contenus des contributions aux colloques CETSIS entre 2001 et 2011, mais des évolutions sont en cours, sans que l'on sache exactement s'il s'agit d'adaptation aux cadres institutionnels qui valorisent les projets tutorés, les projets personnels et professionnels des élèves et des étudiants, ou s'il s'agit de convictions pédagogiques et didactique que des participants plus nombreux chercheraient à mieux étayer.

L'étude des réponses au questionnaire montre que cet outil de professionnalisation (les colloques) tend à poursuivre implicitement des objectifs autres que celui du partage de pratiques d'enseignement. Ainsi, une partie des enquêtés est désireuse de s'orienter vers des pratiques de recherche en didactique et en pédagogie dans le domaine EEA. Le morcellement du domaine en petites spécialités, entre lesquelles les comparaisons de questions didactiques sont pour l'instant absentes, et la tendance à prendre le public étudiant en bloc, comme une donnée contextuelle, montrent la richesse et la difficulté de cette orientation.

Une façon de soutenir l'émergence d'un courant de recherche en didactique et en pédagogie dans le domaine EEA consisterait à ce que les appels à articles et les dispositifs en colloque CETSIS intègrent et prévoient d'une façon plus formelle des textes et des échanges scientifiques consacrés spécifiquement aux analyses didactiques et pédagogiques de dispositifs, de pratiques d'enseignement et d'apprentissages dans les domaines considérés.

\section{Bibliographie}

[1] Georget, J.-P., Thémines, JF., Bailleul, M., "L'enseignement des technologies, des sciences de l'information et des systèmes. Un regard extérieur sur les colloques d'une communauté". Actes du colloque Questions de pédagogie dans l'enseignement supérieur QPES 2013, Sherbrooke (Québec), pp. 235-243. http://www.colloquepedagogie.org/workspaces/colloque_2013/actes_c olloque_qpes2/downloadFile/file/Actes_colloque_ QPES2013.pdf

[2] De Ketele, J.-M., "La pédagogie universitaire : un courant en plein développement". Revue française de pédagogie, $\mathrm{n}^{\circ} 172$, (2010), pp. 5-13.

[3] Granovetter, M., "Economic action and social structure : the problem of embeddedness". American Journal of Sociology, Vol.91, (1985), pp. 481-510.

[4] Matheron, Y., Mémoire et étude des Mathématiques. Une approche didactique à caractère anthropologique. Rennes: Presses universitaires de Rennes (2010).

[5] Wenger, E., Communities of Practice, Learning, Meaning and Identity. Cambridge University Press, (1998). 
[6] Chevallard, J.-Y., "Familière et problématique, la figure du professeur". Recherches en didactique des mathématiques, $\mathrm{n}^{\circ} 17 / 3$, (1997), pp. 17-54

[7] Bonton, P, Coue, E., Mesnard, E., "Préface", Actes du CETSIS-EEA, (2001).

[8] Robbes, D., Jacquet, E., Méry, A., Le Moroux, G., Guillermic, M., Bahna, Z., "Manipulations et matériels partagés Secondaire-Supérieur : une utopie ?", Actes du CETSIS-EEA (2011), Communication $n^{\circ} 29$.

[9] Chevallard, Y., La transposition didactique. Grenoble : La Pensée Sauvage, (1991).

[10]Clot, Y., "De l'analyse des pratiques au développement des métiers", Education et didactique, Vol.1, $\mathrm{n}^{\circ} 1$, pp.83-93.

[11] Sorel, M., Wittorski, R., La professionnalisation en actes et en questions, Paris, L'Harmattan, (2001).

Remerciements : Les auteurs remercient sincèrement les organisateurs du colloque CETSIS 2013 pour leur avoir permis cette recherche innovante, recherche qui a débouché sur un groupe de travail sur les démarche(s) projet à bac \pm 3 et regroupant des acteurs d'horizons variés tant par leur statut que par leur discipline de rattachement. 